CORRECTION

https://doi.org/10.1038/s41586-019-1023-8

\title{
Author Correction: Trade-offs in using European forests to meet climate objectives
}

Sebastiaan Luyssaert, Guillaume Marie, Aude Valade, Yi-Ying Chen, Sylvestre Njakou Djomo, James Ryder, Juliane Otto, Kim Naudts, Anne Sofie Lansø,

Josefine Ghattas \& Matthew J. McGrath

Correction to: Nature https://doi.org/10.1038/s41586-018-0577-1, published online 10 October 2018.

In this Letter, referring to reductions in greenhouse gas emissions in the European Union by 2030 , the statement "About $75 \%$ of this reduction is expected to come from emission reductions and the remaining $25 \%$ from land use, land-use change and forestry" that cited ref. 7 of the original Letter (ref. 1 below) was incorrect. In fact, the 25\% value in ref. 1 refers to global reductions, not those in the European Union. The correct value for the European Union is $1 \%$ (ref. 1), and the text has been corrected accordingly ('75\%' has been changed to '99\%' as well). We thank G. Grassi for identifying the error. Additionally, there were two other minor errors. First, in the sentence "The carbon-sink-maximizing portfolio has a small negative effect on annual precipitation $(-2 \mathrm{~mm})$ and no effect on air temperature (Table 1)" the word 'precipitation' was omitted. Second, Denmark was accidentally deleted during the conversion of Fig. 1. These errors have been corrected online.

1. Grassi, G. et al. The key role of forests in meeting climate targets requires science for credible mitigation. Nat. Clim. Chang. 7, 220-226 (2017). 